\title{
Predicting tablet tensile strength with a model derived from the gravitation-based high-velocity compaction analysis data
}

\section{Tanner, Timo}

2019-07-20

Tanner , T , Antikainen , O , Pollet , A , Räikönen , H, Ehlers , H , Juppo , A \& Yliruusi , J 2019 , ' Predicting tablet tensile strength with a model derived from the gravitation-based high-velocity compaction analysis data ' , International Journal of Pharmaceutics , vol. 566 , pp. 194-202 . https://doi.org/10.1016/j.jpharm.2019.05.024

http://hdl.handle.net/10138/315438

https://doi.org/10.1016/j.jpharm.2019.05.024

cc_by_nc_nd

acceptedVersion

Downloaded from Helda, University of Helsinki institutional repository.

This is an electronic reprint of the original article.

This reprint may differ from the original in pagination and typographic detail.

Please cite the original version. 
1

2

3

4

5

\section{Abstract}

7

8

\title{
Predicting tablet tensile strength with a model derived from the
} gravitation-based high-velocity compaction analysis data

\author{
Tanner Timo, Antikainen Osmo, Pollet Arne, Räikkönen Heikki, Ehlers Henrik,
}

\author{
Juppo Anne, Yliruusi Jouko
}

In the present study, a model was developed to estimate tablet tensile strength utilizing the

gravitation-based high-velocity (G-HVC) method introduced earlier. Three different

formulations consisting of microcrystalline cellulose (MCC), dicalcium phosphate dihydrate (DCP), hydroxypropyl methylcellulose (HPMC), theophylline and magnesium stearate were prepared. The formulations were granulated using fluid bed granulation and the granules were compacted with the G-HVC method and an eccentric tableting machine. Compaction energy values defined from G-HVC data predicted tensile strength of the tablets surprisingly well. It was also shown, that fluid bed granulation improved the compaction energy intake of the granules in comparison to respective physical mixtures. In addition, general mechanical properties and elastic recovery were also examined for all samples. In this study it was finally concluded, that the data obtained by the method was of practical relevance in pharmaceutical formulation development. 


\section{Introduction}

Among pharmaceutical products, tablets are still most widely used. The ease of use, relatively simple manufacture and good shelf-life are some of the benefits of this dosage form. Tablets can be compressed directly from original, physical powder mixtures, but this method is not always feasible, often due to insufficient powder flow. Granulation can improve powder flow, ingredient uniformity and also compression properties in some cases, leading to more successful tablet formulations.

When compressing powder sample inside the die, it undergoes particle rearrangement, fragmentation and deformation (Jain 1999; Jivraj et al. 2000; Mohan 2012). Primarily fragmenting materials are known as brittle. Deformation of the particle can be reversible (elastic) or irreversible (plastic). Under pressure, powder particles deform reversibly until a point known as elastic limit is exceeded. After this point, particles fragment or undergo plastic deformation and after the pressure is lifted, the elastic portion is recovered. While some of these compression mechanics are typically dominant for each material, all materials are capable of undergoing each of these phenomena to some extent. Multiple factors, such as powder particle size, compression speed and water content of the sample, may affect the compression physics of the powder (Amidon \& Houghton 1995; Nokhodchi et al. 1996;

Akande et al. 1997; Sun \& Grant 2001; Nokhodchi 2005). Microcrystalline cellulose is known as a plastic material, starch as an elastic material and dicalcium phosphate as a brittle material, to mention a few (Wu \& Sun 2007; Rashid et al. 2013; Thoorens et al. 2014; Tanner et al. 2018). Excessively elastic formulations may cause defects in tablets, such as capping (Akseli et al. 2013; Furukawa et al. 2015). Contrarily, excessive tablet hardness may result in 
44 insufficient disintegration in the Gl-tract and reduced absorption rate of the active substance (Dor \& Fix 2000; Gupta et al. 2009).

When powder particles are rearranged and packed under pressure, new interparticle bonds are formed and a compact is produced (Jain 1999; Jivraj et al. 2000; Zhang et al. 2003; Li et al. 2004; Patel et al. 2006; Mohan 2012). Bonding mechanisms include, but are not limited to hydrogen bonding, Van Der Waals bonding, solid bridge formation by local melting and mechanical interlocking. Work consumed during particle rearrangement and compact formation is typically quantified by calculating the area under curve of the force-punch displacement plots obtained during tableting (Jain 1999; Antikainen \& Yliruusi 2003; Busignies et al. 2006). This work is generally referred to as compaction energy and may be used as a measure of tablet hardness. Sufficient tensile strength (roughly recommended to be at least $2 \mathrm{MPa}$ ) is an important property of a tablet so that it can withstand stress from further manufacturing unit operations after compaction (Sun et al. 2009; Pitt \& Heasley 2013; Shang et al. 2013; Leane et al. 2015).

In the present work, we utilized our previously developed gravitation-based high-velocity compaction analysis (G-HVC) method (Tanner et al. 2017 \& 2018) to clarify how accurately tablet tensile strength could be predicted by the compaction energy observed during G-HVC measurement. The way the compaction energy is calculated by G-HVC method is novel and is primarily based on the rebound height of the freely falling compressor bar, among some other factors. Without any corrective methods (which may be difficult to refine), the typical area under force-displacement curve includes some errors, including the deformation of steel, which is then included in the result. During G-HVC measurement, the displacement patterns of the compressor bar and the base of the system successfully reveal some of the 
67 error-inducing factors, such as steel deformation. We prepared three different tablet

68 formulations (consisting of five ingredients) with varying microcrystalline cellulose and hydroxypropyl methylcellulose content and granulated them using a fluid bed granulator. The granules were then compressed with the G-HVC method and a tableting machine. Our primary aim was to see if the compaction energy calculated by our method would correlate to the tensile strength of tablets produced by a tableting machine and consequently predict the tablet tensile strength based on the G-HVC data. We also examined the compression mechanics of individual ingredients in their original form and the mixtures, both as physical mixtures and as fluid bed granules.

\section{Materials and methods}

\subsection{Ingredients and powder mixtures}

The ingredients used in the study were microcrystalline cellulose (MCC) (Vivapur 101, JRS Pharma), dicalcium phosphate dihydrate (DCP) (Chemische Fabrik Budenheim), hydroxypropyl methylcellulose (HPMC) (Methocel, DOW Chemical Company), theophylline (Ph.Eur./USP, BASF) and magnesium stearate (Ph.Eur., Yliopiston Apteekki). Three different formulations (PM1-PM3) were mixed and granulated for compression studies (Table 1). Fractions of HPMC and MCC varied between mixtures. True density measurements were performed with a helium pycnometer (Multivolume Pycnometer 1305, Micromeritics Inst. Corp., Norcross, Georgia, USA) and water activity values were measured with Aqualab (Series 3, Decagon Devices Inc., Pullman, Washington, USA). Powder compressions were carried out in a room with controlled temperature and humidity. Controlled temperature 
stayed at $24.7-26.4{ }^{\circ} \mathrm{C}$, humidity at $41.8-46.3 \%$ and water activity of samples at $0.34-0.43$ throughout the study.

\subsection{Fluid bed granulation}

Granulation of the powder mixtures was performed in a fluid bed system (Glatt WSG 5, Glatt $\mathrm{GmbH}$, Binzen, Germany). HPMC was mixed in the formulations and water was used as granulation liquid. Granules were forced through a $1000 \mu \mathrm{m}$-sieve to obtain the batches for the experiment (FBG1-FBG3) (Table 1). The particle size distribution of the granules was measured with in-line particle probe (Parsum IPP 70-SE, Parsum GmbH, Chemnitz, Germany).

\section{Table 1: Powder mixtures (PM=physical mixture; FBG=Fluid bed granules)}

\subsection{Tableting}

Tablets were produced from each granule batch with an instrumented eccentric, singlestation tablet press (Korsch EK 0, Erweka GmbH, Heusenstamm, Germany) with a 9-mm, round and flat-faced punch. Three different force levels were used. For each batch and force level, one hundred tablets were produced and collected sequentially inside transparent plastic tubes so that the properties of selected samples could be compared with the corresponding upper punch pressure during tableting. Twenty tablets were selected from each tube for tensile strength measurement, equalling 180 tablets in total to be measured. Tablet dimensions were measured with a digital micrometer (Sony DZ521, Tokyo, Japan) and 
crushing strength values were measured with a hardness tester (Schleuniger 2E, Dr

Schleuniger Pharmatron AG, Solothurn, Switzerland). This was done to enable studying upper punch pressure-dependent tensile strength correlation for each batch.

\subsection{Gravitational high-velocity compression (G-HVC) measurements}

G-HVC method has been described in our earlier studies (Tanner et al. 2017 \& 2018). In the present study, the weight of the falling compressor bar was $6.27 \mathrm{~kg}$ and the die diameter 8.2 $\mathrm{mm}$. The displacement lasers (Keyence LK-H087, Keyence Corporation of America, Itasca, Illinois, USA) were set at $20 \mathrm{kHz}$ sample rate and $1 \mu \mathrm{m}$ measurement accuracy. The zero point of the displacement measurement was set at the bottom of the empty die so that maximum displacement point and final displacement level depict the height of the sample inside the die. Magnesium stearate-in-acetone ( $5 \% \mathrm{w} / \mathrm{w})$ suspension was applied on the punch before each set of consecutive measurements.

Each sample of individual main ingredients (MCC, DCP, HPMC and theophylline), granules and physical mixtures was compressed five times consecutively, in triplicate. The sample was not removed from the die between the consecutive compressions. Before the first compression, the samples were precompressed by gently lowering the weight on the punch by rotating the adjustment bolt, to induce particle rearrangement without further consolidation. The weight was kept on top of the punch for 60 seconds. This was done to mostly eliminate the rearrangement phase during actual compressions, which is imperative for reliable compaction energy calculations with this method. Sample weights were fixed so that, should the powder reach true density during the measurement, the thickness of the sample would be uniform among all samples. Thus, the sample sizes were affected by the differences in true density. Sample weights were consequently set at $75 \mathrm{mg}$ for MCC and 
theophylline, $65 \mathrm{mg}$ for HPMC, $125 \mathrm{mg}$ for DCP and $85 \mathrm{mg}$ for the mixtures. The initial height for the falling compressor bar was set at $20 \mathrm{~mm}$ distance from the powder bed height after the precompression. Consequently, the kinetic energy of the freely falling bar before the impact was of equal magnitude for all samples, during first compressions of each set. The falling height remained the same throughout later compressions in each set, despite the powder consolidation occurring during the measurement. The differences in the magnitude of powder consolidation were small enough to render height adjustment unnecessary.

\subsection{Compaction energy calculations with the G-HVC method}

When utilizing the G-HVC method, estimating the amount of compaction energy in the sample is primarily based on the rebound height of the falling compressor occurring after the impact. There is no pre-determined displacement pattern for the compressor, unlike in traditional tableting machines. Thus, each sample is allowed to freely resist deformation depending on its properties. The rebound height, too, is ultimately determined by the mechanical properties of the sample.

The total energy input during each compression is the potential energy of the falling compressor, which is determined by the distance between the initial falling height and the maximum displacement point of the compression:

$E_{\text {tot }}=m \times g \times\left(y_{\text {init }}-y_{\text {max }}\right)($ Eq. 1$)$

Where $E_{\text {tot }}=$ total energy input to the system $(\mathrm{J}) ; m=$ weight of the falling compressor $(\mathrm{kg})$; $g=$ gravitational acceleration $\left(\mathrm{m} / \mathrm{s}^{2}\right) ; y_{\text {init }}=$ initial falling height $(\mathrm{m}) ; y_{\max }=$ maximum displacement point $(\mathrm{m})$. 
The zero point of the displacement, $y_{0}$, was set at the bottom of the die and equalled zero.

Consequently, the displacement value depicted the distance between the compressor and the bottom of the die constantly. It is of importance to understand, that the compressor could move freely and depicted the powder bed height only during certain moments, most importantly during maximum displacement point and final displacement level.

The collision between the compressor and the punch is non-elastic, meaning that fraction of the total energy is dissipated during impact. The amount of this non-elastic energy can be determined from the rebound height of the compressor after collision:

$E_{n e}=E_{t o t}-\left(m \times g \times y_{r e b}\right)($ Eq. 2)

Where $E_{n e}=$ total non-elastic energy after collision $(\mathrm{J}) ; y_{r e b}=$ the highest rebounded position of the compressor reached after the collision $(\mathrm{m})$.

During compressions, the resulting non-elastic energy consists of compaction energy of the sample, other sample-originated factors and machine-originated factors. During powder compressions, to determine the machine-originated factors, the base of the system had to be monitored with a second displacement laser (Tanner et al. 2017 \& 2018). When running the machine without powder, a correlation between the energy loss and the maximum velocity of the deformation wave in the base after collision can be found:

$E_{m l}=a \times V_{b \max }^{2}+b \times V_{b \max }($ Eq. 3)

Where $E_{m l}=$ machine-originated energy loss (J); $V_{b \max }=$ highest velocity of the deformation wave observed in the base after collision $(\mathrm{m} / \mathrm{s}) ; a$ and $b=$ resulting constants from correlation determination. After collision, the amount of non-elastic energy during nopowder-compression equals the amount of energy loss which is then compared to the 
deformation wave velocity. To clarify, at this point there was no powder deformation present, so the energy loss was completely related to the method itself.

The correlation can be implemented, when running the machine with powder sample by monitoring the deformation wave in the base. The hypothesis was, that a similar amount of energy is required to cause a similar deformation wave in the base, whether there was powder in the die or not. The machine-originated energy loss factors include, for instance, friction, vibrations, steel-on-steel heat dissipation and sound. The method does not differentiate these factors, but the deformation wave in the base serves as an indicator of the total machine-originated energy loss determined from the rebound height of the compressor, when there is no powder in the die. As discussed in our introductory study, the correlation includes errors as some factors (such as heat) are presumably of different magnitude, depending on whether there is powder in the die or not (Tanner et al. 2017). In this study, machine-originated energy loss correlation was determined by dropping the compressor bar from six different heights (10-60 $\mathrm{mm}$ in $10 \mathrm{~mm}$ increments), in triplicate.

The amount of immediate elastic energy recovered can be directly seen from the displacement data:

$E_{\text {rec }}=m \times g \times\left(y_{\text {final }}-y_{\max }\right)(E q .4)$

Where $E_{r e c}=$ recovered elastic energy in-die immediately after the compression $(\mathrm{J}) ; y_{\text {final }}=$ =final displacement level (m).

In our earlier studies we noticed, that there were also other sample-originated factors contributing to the total energy loss, besides compaction energy (Tanner et al. 2018). Although there was no change in sample consolidation after five compressions, there was 
still detectable internal energy change occurring in the sample. This was assumed to be mainly caused by temporary elastic potential in the sample after the collision. This finding was further supported by noticing that the amount of this internal energy change seemed to increase as the elastic recovery in the sample increased. When powder is compressed under pressure, it undergoes elastic deformation and when the material-dependent yield point (or elastic limit) has been exceeded the material undergoes fragmentation or plastic deformation. During decompression phase, the material recovers its elastic fraction. In our present work, it was assumed that the amount of this elastic energy stayed similar throughout five compressions (or in other words, the elastic limit of the material remained unchanged). The prerequisite for this assumption was, that the rearrangement phase had to be eliminated from the first compression by precompressing the sample. Otherwise, the amount of elastic energy could have been less than expected, due to amount of energy consumed during powder rearrangement. Also, the sample must have consolidated to the point where there was no more permanent deformation occurring, after the five compressions. Therefore, the remaining non-elastic energy detected after the fifth compression, $E_{s l}$, equals the other sample-originated energy loss besides compaction energy:

$E_{s l}=E_{n e 5}-E_{m / 5}-E_{\text {rec5 }}($ Eq. 5)

Where $E_{\text {nes}}=$ total non-elastic energy after collision during fifth compression (J); $E_{m / 5}=$ machine-originated energy loss during fifth compression (J); $E_{\text {rec5 }}=$ recovered elastic energy immediately after the fifth compression $(\mathrm{J}) . E_{s l}$ is determined from the fifth compression and is utilized as a constant throughout all compressions within a set. 
221 Finally, the compaction energy bound in the sample, $E_{c o m p}$, is determined by subtracting the

222 other factors from the total non-elastic energy observed:

$223 E_{c o m p}=E_{n e}-E_{m l}-E_{r e c}-E_{s l}($ Eq. 6)

224 The equation is adjusted so that the compaction energy after the fifth compression, $E_{c o m p 5}$, 225 equals zero:

$226 E_{c o m p 5}=E_{n e 5}-E_{m 15}-E_{r e c 5}-E_{s l}$

$227 \quad E_{s l}=E_{n e 5}-E_{m / 5}-E_{\text {rec5 }} ;$

$228 E_{c o m p 5}=E_{s l}-E_{s l}=0$ (Eq. 7)

229 Relationship between the compaction energy and the sample size can be shown as:

$230 \quad E_{c o m p s}=E_{c o m p} / w_{s}$ (Eq. 8)

231 Where $E_{c o m p s}=$ specific compaction energy $(\mathrm{J} / \mathrm{g}) ; \mathrm{w}_{\mathrm{s}}=$ sample weight $(\mathrm{g})$.

232 In summary, to determine the compaction energy in the sample with the current method,

233 machine-originated factors, elastic recovery and other sample-originated factors are

234 subtracted from the total non-elastic energy. In this current work, the compaction energy

235 during fifth compression is always considered zero. It is required that before the first

236 compression, the rearrangement phase has been substantially eliminated through

237 precompression and that there is no more consolidation occurring after the fifth

238 compression.

239 2.6. Machine deformation and powder elastic recovery estimation

240 When estimating immediate in-die axial elastic recovery of the samples, the machine

241 deformation had to be accounted for. This was determined from the same data as the 
242

243

machine-originated energy loss, where the compressor was dropped without powder in the die. When the zero point of the displacement laser was set at the bottom of the die, negative distance values were obtained during collision, due to machine deformation. The magnitude of this value is force-dependent, so a correlation could be made:

$y_{\text {mach }}=c \times F^{2}+d \times F($ Eq. 9)

Where $y_{\text {mach }}=$ absolute value of axial machine deformation $(\mathrm{m})$; $F=$ compression force $(\mathrm{N})$; $c$ and $d=$ resulting constants from correlation determination.

Therefore, immediate in-die axial elastic recovery of the sample, el $l_{\text {iia }}(\%)$, is calculated as:

$e l_{\text {iia }}=100 \times\left(y_{\text {final }}-\left(y_{\max }+y_{\text {mach }}\right)\right) / y_{\text {final }}($ Eq. 10)

\subsection{MATLAB derivations}

G-HVC program was coded with MATLAB software (version R2018a, Mathworks Inc, Natick, Massachusetts, USA). The settings and the principle of the program were same as in our previous study (Tanner et al. 2018). Savitzky-Golay derivation (second-order polynomial fit, window size 21) was used to obtain velocity from the displacement data. Velocity data was smoothed with a sigmoidal fit to obtain the second derivative, acceleration. Due to sigmoidal fit, acceleration data required no more filtering. Compression force and pressure could be directly calculated from the acceleration data, since the weight of the compressor and the surface area of the punch were known. 


\subsection{Granule particle size distribution}

Particle size distribution of granules (FBG1-FBG3) can be seen in Table 2. Some of the original granule batches contained very large particles, so the sieving was carried out to decrease the possible disturbance caused by them. Particles too large may be problematic during the tableting process in many ways, for instance, by decreasing the available contact area between particles or by affecting the final product through uneven content uniformity (Rohrs et al. 2006; Virtanen et al. 2010). Also, increase in particle size may possibly enhance the fragmentation of the sample (Sun \& Grant 2001; Patel et al. 2007). Granule particle size increased with increasing HPMC content, which was to be expected (Planinšek et al. 2000; Chitu et al. 2011).

\subsection{Tensile strength of tablets}

During tableting, even if the machine settings are kept constant, there is variation in upper punch pressure, depending on the amount of powder inside the hopper and other factors affecting powder flow, such as, machine vibration. Therefore, tablets were collected inside tubes so that the measured tensile strength value could be compared to the corresponding upper punch pressure for each tablet (Fig. 1). All batches showed good correlation between the upper punch pressure and the resulting tensile strength. Due to differences in pressure at the highest force level, the overall results of this work could only be compared up to 200 
$\mathrm{MPa}$, to avoid the possible errors caused by extrapolation. It was assumed, that differences in deformation resistance of the material during tableting caused variation in the compaction pressure. Consequently, there is some variation between the force levels, even though the predetermined tableting parameters were kept constant. FBG3, which contained the highest fraction of HPMC and the lowest fraction of MCC, showed lowest tensile strength of all batches. $F B G 1$ and $F B G 2$ showed no significant difference between them. The sudden change in tabletability of the mixture, when the MCC content drops from 68 to $58 \%$ (from $F B G 2$ to $F B G 3$ ), can be explained by percolation theory (Kuentz \& Leuenberger 2000; Amin \& Fell 2004). It was reasonable to assume, that a percolation threshold existed between FBG3 and the rest of the mixtures. It is essential to add, that powder flow was sufficient during tableting and the tablet weight within all batches only varied between 255 and 266 mg. Furthermore, the weight variation was random and no trends indicating exceptional die filling for any specific batch was detected.

Fig. 1: The correlation between the upper punch pressure and the tensile strength of the tablets.

\subsection{Machine-originated energy loss and machine deformation correlations for G-HVC measurements}

The correlations concerning machine-originated energy loss and machine deformation can be seen in Fig. $2 \& 3$. The correlation for machine-originated energy loss, $E_{m l}$, was expectedly very similar to our previous studies (Fig. 2). When the machine was being run with powder 
in the die, the maximum velocity of the deformation wave in the base was utilized as an indicator for the energy loss according to this correlation. The machine-originated factors were assumed to be similarly force-dependent, whether there was powder inside the die or not. This assumption contained a small error, since it was possible that the relative amount of some factors, such as heat dissipation, could have occurred differently when the powder sample was present. The correlation was considered accurate enough for the present work.

\section{Fig. 2: Machine-originated energy loss during G-HVC measurements, determined from} compressions without powder sample $(n=3)$.

\section{When estimating the displacement due to machine deformation, the results randomly} showed some deviation from the trendline (Fig. 3). This was assumed to occur due to small position changes in the base of the system between compressions. According to this hypothesis, when the surfaces of the compressor and the punch were facing each other without any contact angle, the system allowed more deformation during collision. The correlation was considered to be accurate enough for this study and the phenomenon was already expected to occur based on our previous studies. It is of importance to understand that $y_{\text {mach }}$ determined here refers to all machine deformation and not only the deformation of the punch. Without thorough analysis and study of its own, it is impossible to determine specifically which parts of the system deform and to what degree.

\section{Fig. 3: Machine deformation during G-HVC measurements without powder sample $(n=3)$.}


Porosity change after five consecutive compressions for the mixtures and individual main ingredients can be seen in Fig. 4. Small amount of lubricant was considered a necessary ingredient for any formulation and therefore magnesium stearate was not specifically studied in this work. It is generally known that excessive amount of magnesium stearate in a formulation produces weaker tablets (Aoshima et al. 2005; Late et al. 2009; Koskela et al. 2018). In the present study, the amount of magnesium stearate in the mixtures was reasonable.

MCC and DCP showed similar compression mechanics as in our earlier works (Fig. 4A). MCC showed steady decrease in porosity which implied plastic behaviour. DCP showed small change in porosity and remained most porous of all ingredients. It was already known, that the compression pressure in our setup would not exceed the high yield point of fragmenting DCP, which is reported to be over 300 MPa (Doldán et al. 1995; Nicklasson et al. 2009). Presumably, instead of extensive destruction of the particles, fragmentation for DCP only occurred at high-stress contact points. HPMC reached a steady level at around $10 \%$ porosity which is logical due to its high elastic recovery (which is discussed further later in the text). 
351

352

negative porosity was already reached after the first compression. It has been reported that theophylline deforms plastically and shows excellent compressibility (Suihko et al. 2001; Chattoraj et al. 2010; Chang \& Sun 2017). However, the porosity values shown were based on true density values obtained from helium pycnometer (Table 3). It is possible, that during helium pycnometry measurement, some of the pores inside the particles may be unmeasurable or the loosely bound water content of the sample may distort the result (Viana 2002; Sun 2004; Sun 2005). Consequently, the measured volume is too high and true density value too low. True density of theophylline is reported to be roughly $1.5 \mathrm{~g} / \mathrm{cm}^{3}$, which is higher than the value obtained in the present work, as seen in Table 3 (Viana 2002; Sun 2004; Hadžović et al. 2010). This could explain the remarkable negative value obtained. However, even if the value 1.5 was used instead of the measured value, the porosity of theophylline would still reach negative values. MCC and HPMC could also temporarily reach negative porosity during the maximum displacement point which could also be explained by the type of true density measurement. While theophylline could be susceptible to polymorphic changes, it has also been shown not to occur during compaction (Suihko et al. 2001; Hadžović et al. 2010). Wet granulation process (with hydroxypropyl cellulose, HPC), on the other hand, has been reported to affect the structure of theophylline, namely the transition to amorphous form (Sakamoto et al. 2016). However, the individual ingredients were not granulated in this work, while studying them with G-HVC method. For the aims of this work, the specific reason for the negative porosity of theophylline is not discussed further. Also, the small amount of theophylline served as a marker ingredient and it presumably affected the results very little, when mixtures were being examined. 
In Fig. 4B-4D, the differences between physical mixtures and granules seemed rather subtle. The graphs mostly differ at the first compression and after that start resembling each other. Formulations 1 and 3 seemed to consolidate more in granular form compared to their physical mixture forms after all compressions but according to Student's t-test, there is no significant difference between the results. The most significant difference between the physical mixtures and granules was that the porous fluid bed granules were not as consolidated after the first compression as their respective physical mixtures for Formulations 2 and 3. The fluid bed granules were more porous to begin with and therefore it was not surprising that there were differences in consolidation after first compressions.

In-die axial elastic recovery of the samples after the first compression of each set is depicted in Fig. 5. MCC and DCP behaved similarly as in our earlier studies (Tanner et al. 2017 \& 2018). HPMC showed remarkable elastic recovery, comparable to that of Starch 1500 in our previous study. This is interesting, since HPMC is used as a binder. Despite its binding effect, excessive amount could produce weaker tablets due to its high elastic recovery. This could have been the reason for tablets made of FBG3-granules having the lowest tensile strength in the present work (Fig. 1). Plastic theophylline showed very little elastic recovery, which further contributed to the excellent compressibility of the material. The obtained elastic recovery values of the individual ingredients were in agreement with the existing literature

394 (Picker 2001; Hardy et al. 2006; Chattoraj et al. 2010; Haware et al. 2010; Chang \& Sun 2017). It is of importance to understand, that in the present work, only immediate in-die 
396

397

axial elastic recovery was studied. The elastic recovery values could have been higher, had the samples been removed from the die.

Elastic recovery of physical mixtures and the corresponding granules did not seem to differ greatly (Fig. 5). The mixtures contained mostly HPMC and MCC which explained their overall elastic recovery values being between 10 and $15 \%$. It was slightly unexpected that Formulation 3 did not appear more elastic than the rest of the mixtures even though it contained the largest amount of HPMC. It has been reported that wet granulation may cause changes in MCC structure, for instance, hardening of the material, which could presumably affect elastic recovery (Parker et al. 1991; Suzuki et al. 2001; Badawy et al. 2006). It is also possible that within all mixtures, the large amount of MCC dominates the mechanical properties according to the percolation theory and encapsulates the elastic HPMC in its structure. This could also mean, that the compacts made of FBG3-granules contained more stored elastic potential within them after the compressions, when compared to rest of the mixtures. This phenomenon could cause problems later, as the elastic portion could recover slowly during storage, possibly fracturing the compact. In the present work, however, this phenomenon could not be confirmed by these results alone.

Fig. 5: Immediate in-die axial elastic recovery of samples during the first compression of each set ( $n=3$; average \pm standard deviation). 


\subsection{Compaction energy determined by G-HVC method}

419 By adjusting the sample sizes depending on their true density, the total energy input for all

420 the compressions only varied between 1.25 and $1.35 \mathrm{~J}$. Specific compaction energy values

421 for the individual ingredients can be seen in Fig. 6. The fifth compressions were omitted, due to the assumption that there was no more consolidation occurring during the fifth compression and the compaction energy value equalled zero in all cases. Most energy in total was bound to MCC of all samples. The energy intake for theophylline was initially high, but it drastically decreased during later compressions. It is of importance to note that due to high density of DCP (and consequently greater sample size in this work), the amount of specific compaction energy in joules per gram was lowest for DCP. Similarly, HPMC showed surprisingly high energy intake, since its sample size was lowest. It has been reported that MCC shows generally higher values of compaction energy in joules per gram when compared to DCP (Coffin-Beach \& Hollenbeck 1983; Aburub et al. 2007; Michaut et al. 2010). This was also apparent in our earlier work (Tanner et al. 2018). (Studies comparing HPMC or theophylline to MCC or DCP were not found.) Having uniform sample size could solve the issue of having to explain some of the differences in the results, but for the present work, having uniform total energy input was considered more important. Furthermore, the sample sizes were uniform among the study of mixtures, which was the main object of interest in this work. 
441 Comparing energy intake in this manner could be important, when approximating the

442 tabletability of precompressed materials and mixtures. Again, the precompression in this

443 study was done by gently lowering the compressor bar on top of the sample to cause

444 rearrangement in the sample, which did not cause huge impact on the sample. However,

445 when tablets are produced with a rotary tableting machine or when the physical mixture is

446 granulated from a precompressed ribbon, the amount of bound energy during

447 precompaction can be substantial enough to affect the tabletability of the mixture (Patel et

448 al. 2011; Sun \& Kleinebudde 2016; Grote \& Kleinebudde 2018). As results in Fig. 6 showed,

449 theophylline lost a substantial amount of its energy intake potential between the first and

450 second compression. Based on this result, one could exercise caution when precompressing

451 theophylline, to prevent the loss of tabletability. MCC, on the other hand, showed

452 preferable behaviour for precompression as the difference between first and subsequent

453 compression was smaller.

454 The compaction energy results for the mixtures can be seen in Fig. 7. The results clearly

455 pointed that the total compaction energy for fluid bed granules was generally higher than

456 that of the respective physical mixture. This was not entirely surprising, since fluid bed

457 systems and wet granulation in general may enhance the tabletability of the mixture (Li \&

458 Peck 1990; Arndt et al. 2018). The results for Formulations 1 and 2 seemed quite similar,

459 while Formulation 3 showed lower energy intake. 
463

464

465

466

467

\subsection{Compaction energy of granule mixtures vs. tablet tensile strength}

The primary aim of this study was to see if the compaction energy value obtained for the granules by G-HVC method could predict the tensile strength of the tablets made of the same granules. Since the falling compressor bar is slowed down in a unique manner, depending on the properties of powder, the compression pressure varied between different samples (and also between repetitions for a sample). The compression pressure for G-HVC was deliberately set at a lower level than that of the tableting machine and varied between 40 and $64 \mathrm{MPa}$ during first compressions. When comparing these numbers to the results from tableting (Fig. 1), it could be seen that a coherent tablet was barely produced during the first compression in G-HVC measurement. Despite this, we aimed to see whether the differences in the compaction energy intake of the samples, even when pressure is low, would predict the strength of tablets produced with a tableting machine. Also, the sample size in G-HVC measurements was smaller ( $85 \mathrm{mg}$ ) than that during the tableting experiment (260 mg). Lower amount of particle contact points assumedly increased the pressure per contact point during G-HVC measurements, allowing the materials to yield as seen in Fig. 4.

According to Fig. 1, between 100 and $200 \mathrm{MPa}$ compression pressure, the tensile strength of tablets varied roughly between 2 and $4 \mathrm{MPa}$. These two compression pressure values were chosen for the models, since all granule mixtures could be then compared without extrapolation. The model predicting tablet tensile strength can be seen in Fig. 8. In this model, only the first compression of each G-HVC-set was considered relevant. During G-HVC measurements, there was also naturally variation in compression pressure. Therefore, in this model, the lowest pressure value obtained during repetitions of the sample was set at 100 or $200 \mathrm{MPa}$ and the rest of the repetitions obtained relatively same pressure variation 
as in G-HVC measurements. The model showed good correlation between the compaction energy and tensile strength.

Fig 8: The correlation between compaction energy obtained from the first compression of each set determined by G-HVC method and the tensile strength of the tablets produced with the tableting machine. A) $100 \mathrm{MPa}$ model pressure; B) $200 \mathrm{MPa}$ model pressure

It can be stated, that in this study, the determination of compaction energy using the G-HVC method was accurate enough to predict the tensile strength of the tablets. Based on G-HVC results alone, one could have discarded $F B G 3$, had the aim been to produce tablets with higher tensile strength (Fig. 8). There were no remarkable differences between FBG1 and FBG2 and the same result was obtained from both, G-HVC measurements and actual tableting. It is of importance to clarify, that the selection of ingredients and their fractions were only considered for their mechanical properties. Their pharmacological or biopharmaceutical functions were not relevant for this study.

\subsection{Other general discussion}

For the tensile strength prediction to succeed by this method, one major issue has to be considered. Low pressure was deliberately used during G-HVC measurements to show whether the material characteristics would predict the energy intake ability and subsequent tablet hardness after compaction with a tableting machine. The material or mixture, however, should not presumably show any drastic differences in their compression mechanics within this pressure range. For instance, had we studied a fragmenting material 
with a yield pressure point somewhere between the G-HVC pressure and actual tableting pressure, major errors could have occurred. It is reasonable to suggest, that the components of the mixture should yield with both pressure levels (during G-HVC and tableting). In our study, we showed that all individual materials consolidated during G-HVC as seen in Fig. 4. Furthermore, coherent compacts were produced from all samples during G-HVC measurements. Therefore, it was assumed that the compression mechanics of the ingredients were not significantly different during G-HVC measurements or actual tableting. One might also argue that the compression pressure during G-HVC should be set at a higher level by default. However, to obtain accurate results with the method, the tooling must not undergo any amount of deformation that is not recovered immediately during the measurement window. We have examined higher pressure levels during G-HVC, only to see that steel can rather easily undergo the type of deformation, that takes more than seconds to recover.

This study alone is not sufficient to prove that the compaction energy would always correlate similarly to tensile strength. For instance, having only fragmenting materials in the mixture could give completely different energy values compared to the plastic-elastic compositions depending on which of the bonding phenomena would be dominantly present. One might assume, that the compaction energy could contribute differently to the tensile strength, if the main type of bonding was, for instance, hydrogen bonding instead of Van der Waals bonding, or other way around. It is also important to remember that decent tensile strength is only one of many requirements for a good tablet.

The results of this work encourage studying G-HVC method further as an aid in screening for the feasible formulation, primarily during the early stages of pharmaceutical development. 
531
The setup is rather inexpensive and small in size when compared to tableting machines. Individual samples can be quickly measured with high accuracy. Compaction energy is calculated in a novel and unique manner with the G-HVC method. Machine deformation and other factors affecting energy dissipation during compression have been considered in compaction energy calculations, providing accurate and reproducible results. All data is solely based on the displacement patterns of the compressor and the base of the system without the need for excessive amount of various equipment. Also, the properties of each powder sample ultimately determine the displacement profile, providing unique data for each examined sample even if the differences between their mechanical properties were minor.

\section{Conclusions}

In conclusion, G-HVC method was successfully utilized to correlate with differences in tablet tensile strength for three different fluid bed granule formulations, with varying MCC and HPMC content. One could have correctly discarded the formulation with the highest HPMC content based on G-HVC results alone, had the aim been to produce tablets with higher tensile strength. Compaction energy, calculated in a novel manner with the method, showed good correlation with the tensile strength of the corresponding tablets. Porosity, elastic recovery and compaction energy for both mixtures and individual ingredients were also examined. All data was solely based on the distance-time data, which was unique for each powder sample. Even minor differences seen in the G-HVC data, reflected the results from actual tableting, proving the method to be applicable in practical pharmaceutical formulation development. 


\section{Conflicts of interest}

556 None

557

\section{Acknowledgements}

559 This paper is dedicated to emeritus professor Jouko Yliruusi's remarkable career as a

560 pharmacist, teacher, supervisor and researcher. His boundless imagination has inspired

561 many and his impact in the field of pharmacy is too large to measure. His positive and easy-

562 going attitude has given strength to us all, his students and colleagues. With gratitude, we

563 wish him peaceful days of retirement. Yet knowing him, the work is not over.

564 Also, we would like to kindly acknowledge Tuomas Saarinen, Robin De Corte and Visa

565 Mattila for collaboration.

566 This research did not receive any specific grant from funding agencies in the public,

567 commercial, or not-for-profit sectors.

568

569

570

571

572 
574

575

576

577

578

579

580

581

582

583

584

585

586

587

588

589

590

591

592

593

594

\section{References}

Aburub, A., Mishra, D., Buckner, I., 2007. Use of compaction energetics for understanding particle deformation mechanism. Pharm. Dev. Technol. 12, 405-414.

Akande, O.F., Rubinstein, M.H., Rowe, P.H., Ford, J.L., 1997. Effect of compression speeds on the compaction properties of a 1:1 paracetamol-microcrystalline cellulose mixture prepared by single compression and by combinations of pre-compression and main-compression. Int. J. Pharm. 157, 127-136.

Akseli, I., Ladyzhynsky, N., Katz, J., He, X., 2013. Development of predictive tools to assess capping tendency of tablet formulations. Powder Technol. 236, 139-148.

Amidon, G.E., Houghton, M.E., 1995. The effect of moisture on the mechanical and powder flow properties of microcrystalline cellulose. Pharm. Res 12, 923-929.

Amin, M.C.I., Fell, J.T., 2004. Comparison studies on the percolation thresholds of binary mixture tablets containing excipients of plastic/brittle and plastic/plastic deformation properties. Drug Dev. Ind. Pharm. 30, 937-945.

Antikainen, O., Yliruusi, J., 2003. Determining the compression behaviour of pharmaceutical powders from the force-distance compression profile. Int. J. Pharm. 252, 253-261.

Aoshima, H., Miyagisnima, A., Nozawa, Y., Sadzuka, Y., Sonobe, T., 2005. Glycerin fatty acid esters as a new lubricant of tablets. Int. J. Pharm. 293, 25-34.

Arndt, O.-R., Baggio, R., Adam, A.K., Harting, J., Franceschinis, E., Kleinebudde, P., 2018. Impact of different dry and wet granulation techniques on granule and tablet properties: A comparative study. J. Pharm. Sci. 107, 3143-3152. 
595

596

597

598

599

600

601

602

603

604

605

606

607

608

609

610

611

612

613

614

615

Badawy, S.I.F., Gray, D.B., Hussain, M.A., 2006. A study on the effect of wet granulation on microcrystalline cellulose particle structure and performance. Pharm. Res. 23, 634-640.

Busignies, V., Leclerc, B., Porion, P., Evesque, P., Couarraze, G., Tcholeroff, P., 2006.

Compaction behaviour and new predictive approach to the compressibility of binary mixtures of pharmaceutical excipients. Eur. J. Pharm. Biopharm. 64, 66-74.

Chang, S.-Y., Sun, C.C., 2017. Superior plasticity and tabletability of theophylline monohydrate. Mol. Pharm. 14, 2047-2055.

Chattoraj, S., Shi, L., Sun, C.C., 2010. Understanding the relationship between crystal structure, plasticity and compaction behaviour of theophylline, methyl gallate, and their 1:1 co-crystal. CrystEngComm 12, 2466-2472.

Chitu, T.M., Oulahna, D., Hemati, M., 2011. Wet granulation in laboratory scale high shear mixers: Effect of binder properties. Powder Technol. 206, 25-33.

Coffin-Beach, D.P., Hollenbeck, R.G., 1983. Determination of the energy of tablet formation during compression of selected pharmaceutical powders. Int. J. Pharm. 17, 313-324.

Doldán, C., Souto, C., Concheiro, A., Martínez-Pacheco, R., Gómez-Amoza, J.L., 1995.

Dicalcium phosphate dihydrate and anhydrous dicalcium phosphate for direct compression: a comparative study. Int. J. Pharm. 124, 69-74.

Dor, P.J.M., Fix, J.A., 2000. In vitro determination of disintegration time of quick-dissolve tablets using a new method. Pharm. Dev. Technol. 5, 575-577.

Furukawa, R., Chen, Y., Horiguchi, A., Takagaki, K., Nishi, J., Konishi, A., Shirakawa, Y., Sugimoto, M., Narisawa, S., 2015. Numerical evaluation of the capping tendency of 
616

617

618

619

620

621

622

623

624

625

626

627

628

629

630

631

632

633

634

635

microcrystalline cellulose tablets during a diametrical compression test. Int. J. Pharm. 493, 182-191.

Grote, S., Kleinebudde, P., 2018. Roll compaction/dry granulation of dibasic calcium phosphate anhydrous-Does the morphology of the raw material influence the tabletability of dry granules? J. Pharm. Sci. 107, 1104-1111.

Gupta, A., Hunt, R.L., Shah, R.B., Sayeed, V.A., Khan, M.A., 2009. Disintegration of highly soluble immediate release tablets: a surrogate for dissolution. AAPS PharmSciTech 10, 495499.

Hadžović, E., Betz, G., Hadžidedić, Š., El-Arini, S.K., Leuenberger, H., 2010. Roller compaction of different pseudopolymorphic forms of theophylline: Effect on compressibility and tablet properties. Int. J. Pharm. 396, 53-62.

Hardy, I.J., Cook, W.G., Melia, C.D., 2006. Compression and compaction properties of plasticised high molecular weight hydroxypropylmethylcellulose (HPMC) as a hydrophilic matrix carrier. Int. J. Pharm. 311, 26-32.

Haware, R.V., Tho, I., Bauer-Brandl, A., 2010. Evaluation of a rapid approximation method for the elastic recovery of tablets. Powder Technol. 202, 71-77.

Jain, S., 1999. Mechanical properties of powders for compaction and tableting: an overview. Pharm. Sci. Technol. Today 2, 20-31.

Jivraj, M., Martini, L.G., Thomson C.M., 2000. An overview of the different excipients useful for the direct compression of tablets. Pharm. Sci. Technol. Today 3, 58-63. 
Koskela, J., Morton, D.A.V., Stewart, P.J., Juppo, A.M., Lakio, S., 2018. The effect of mechanical dry coating with magnesium stearate on flowability and compactibility of plastically deforming microcrystalline cellulose powders. Int. J. Pharm. 537, 64-72.

Kuentz, M., Leuenberger, H., 2000. A new theoretical approach to tablet strength of a binary mixture consisting of a well and a poorly compactable substance. Eur. J. Pharm. Biopharm. 49, 151-159. Late, S.G., Yu, Y.-Y., Banga, A.K., 2009. Effects of disintegration-promoting agent, lubricants and moisture treatment on optimized fast disintegrating tablets. Int. J. Pharm. 365, 4-11. Group, 2015. A proposal for a drug product Manufacturing Classification System (MCS) for oral solid dosage forms. Pharm. Dev. Technol. 20, 12-21. flowability and compactibility. Int. J. Pharm. 280, 77-93. properties of a maltodextrin product, Maltrin 150 ${ }^{\text {TM }}$. Drug Dev. Ind. Pharm. 16, 1491-1503. 2010. Evaluation of a rotary tablet press simulator as a tool for the characterization of compaction properties of pharmaceutical products. J. Pharm. Sci. 99, 2874-2885. 
656

657

658

659

660

661

662

663

664

665

666

667

668

669

670

671

672

673

674

675

676

677

Nicklasson, F., Johansson, B., Alderborn, G., 1999. Occurrence of fragmentation during compression of pellets prepared from a 4 to 1 mixture of dicalcium phosphate dihydrate and microcrystalline cellulose. Eur. J. Pharm. Sci. 7, 221-229.

Nokhodchi, A., Ford, J.L., Rowe, P.H., Rubinstein, M.H., 1996. The effects of compression rate and force on the compaction properties of different viscosity grades of hydroxypropylmethylcellulose 2208. Int. J. Pharm. 129, 21-31.

Nokhodchi, A., 2005. An overview of the effect of moisture on compaction and compression. Pharm. Technol. 29, 46-66.

Parker, M.D., York, P., Rowe, R.C., 1991. Binder-substrate interactions in wet granulation. 2: The effect of binder molecular weight. Int. J. Pharm. 72, 243-249.

Patel, S., Kaushal, A.M., Bansal, A.K., 2006. Compression physics in the formulation development of tablets. Crit. Rev. Ther. Drug Carrier Syst. 23, 1-65.

Patel, S., Kaushal, A.M., Bansal, A.K., 2007. Effect of particle size and compression force on compaction behavior and derived mathematical parameters of compressibility. Pharm. Res. 24, 111-124.

Patel, S., Dahiya, S., Sun, C.C., Bansal, A.K., 2011. Understanding size enlargement and hardening of granules on tabletability of unlubricated granules prepared by dry granulation. J. Pharm. Sci. 100, 758-766.

Picker, K.M., 2001. Time dependence of elastic recovery for characterization of tableting materials. Pharm. Dev. Technol. 6, 61-70.

Pitt, K.G., Heasley, M.G., 2013. Determination of the tensile strength of elongated tablets. Powder Technol. 238, 169-175. 
678

679

680

681

682

683

684

685

686

687

688

689

690

691

692

693

694

695

696

697

698

Planinšek, O., Pišek, R., Trojak, A., Srčič, S., 2000. The utilization of surface free-energy parameters for the selection of a suitable binder in fluidized bed granulation. Int. J. Pharm. $207,77-88$.

Rashid, I., Mahmoud, M.H., Omari, A., Badwan, A.A., 2013. From native to multifunctional starch-based excipients designed for direct compression formulation. Starch 65, 552-571.

Rohrs, B.R., Amidon, G.E., Meury, R.H., Secreast, P.J., King, H.M., Skoug, C.J., 2006. Particle size limits to meet USP content uniformity criteria for tablets and capsules. J. Pharm. Sci. 95, 1049-1059.

Sakamoto, T., Sasaki, T., Katori, N., Goda, Y., 2016. Analysis of pseudo-polymorphism conversion of theophylline during wet granulation and drying processes and effect of binder on dehydration and amorphization. J. Infrared Millim. Terahertz Waves 37, 1007-1020.

Shang, C., Sinka, I.C., Jayaraman, B., Pan, J., 2013. Break force and tensile strength relationships for curved faced tablets subject to diametrical compression. Int. J. Pharm. 442, 57-64.

Suihko, E., Lehto, V.-P., Ketolainen, J., Laine, E., Paronen, P., 2001. Dynamic solid-state and tableting properties of four theophylline forms. Int. J. Pharm. 217, 225-236.

Sun, C., Grant, D.J.W., 2001. Effects of initial particle size on the tableting properties of Llysine monohydrochloride dihydrate powder. Int. J. Pharm. 215, 221-228.

Sun, C.C., 2004. A novel method for deriving true density of pharmaceutical solids including hydrates and water-containing powders. J. Pharm. Sci. 93, 646-653.

Sun, C.C., 2005. True density of microcrystalline cellulose. J. Pharm. Sci. 94, 2132-2134. 

drug load tablet formulation based on assessment of powder manufacturability: moving towards quality by design. J. Pharm. Sci. 98, 239-247.

Sun, C.C., Kleinebudde, P., 2016. Mini review: Mechanisms to the loss of tabletability by dry granulation. Eur. J. Pharm. Biopharm. 106, 9-14. characteristics of microcrystalline cellulose during wet granulation using a high-shear mixer. J. Pharm. Pharmacol. 53, 609-616. high-velocity compaction analysis method for pharmaceutical powders. Int. J. Pharm. 526, 31-40.

Tanner, T., Antikainen, O., Ehlers, H., Blanco, D., Yliruusi, J., 2018. Examining mechanical properties of various pharmaceutical excipients with the gravitation-based high-velocity compaction analysis method. Int. J. Pharm. 539, 131-138. direct compression binder in a quality by design environment-a review. Int. J. Pharm. 473, $64-72$.

716 Viana, M., Jouannin, P., Pontier, C., Chulia, D., 2002. About pycnometric density 717 measurements. Talanta 57, 583-593.

718 Virtanen, S. Antikainen, O., Räikkönen, H., Yliruusi, J., 2010. Granule size distribution of 719 tablets. J. Pharm. Sci. 99, 2061-2069. 
720 Wu, S-J., Sun, C.C., 2007. Insensitivity of compaction properties of brittle granules to size

721 enlargement by roller compaction. J. Pharm. Sci. 96, 1445-1450.

722 Zhang, Y., Law, Y., Chakrabarti, S., 2003. Physical properties and compact analysis of

723 commonly used direct compression binders. AAPS PharmSciTech 4, 489-499.

724 
Table 1

\begin{tabular}{|l|l|l|l|l|l|}
\hline & \multicolumn{3}{|l|}{ Meight-\% } & \multicolumn{2}{l|}{} \\
& MCC & DCP & HPMC & Theophylline & Magn.stear. \\
\hline Formulation 1: & 73 & 20 & 5 & 1 & 1 \\
\hline PM1/FBG1 & & & & & \\
\hline PM2/FBG2 & 68 & 20 & 10 & 1 & 1 \\
\hline Formulation 3: & & & & & \\
\hline
\end{tabular}

Table 2

\begin{tabular}{|cccc|}
\hline & $d 10(\mu \mathrm{m})$ & $d 50(\mu \mathrm{m})$ & $\mathrm{d} 90(\mu \mathrm{m})$ \\
FBG1 & $91 \pm 1$ & $292 \pm 6$ & $481 \pm 8$ \\
FBG2 & $94 \pm 3$ & $497 \pm 14$ & $788 \pm 20$ \\
FBG3 & $266 \pm 3$ & $639 \pm 5$ & $984 \pm 21$ \\
\hline
\end{tabular}

728

Table 3

\begin{tabular}{|cc|}
\hline & True density $\left(\mathrm{g} / \mathrm{cm}^{3}\right)$ \\
MCC & $1.439 \pm 0.002$ \\
DCP & $2.365 \pm 0.014$ \\
HPMC & $1.233 \pm 0.005$ \\
Theophylline & $1.382 \pm 0.005$ \\
Magnesium stearate & $1.026 \pm 0.008$ \\
\hline
\end{tabular}


Fig. 1

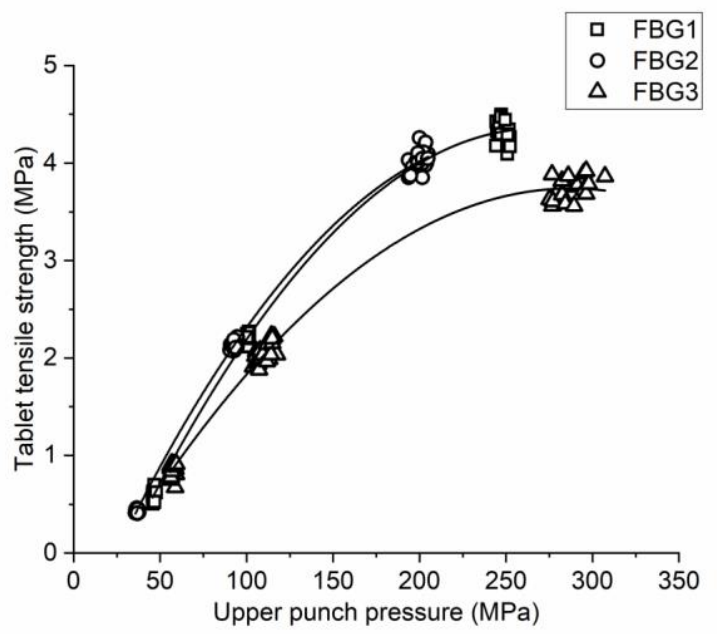

Fig. 2

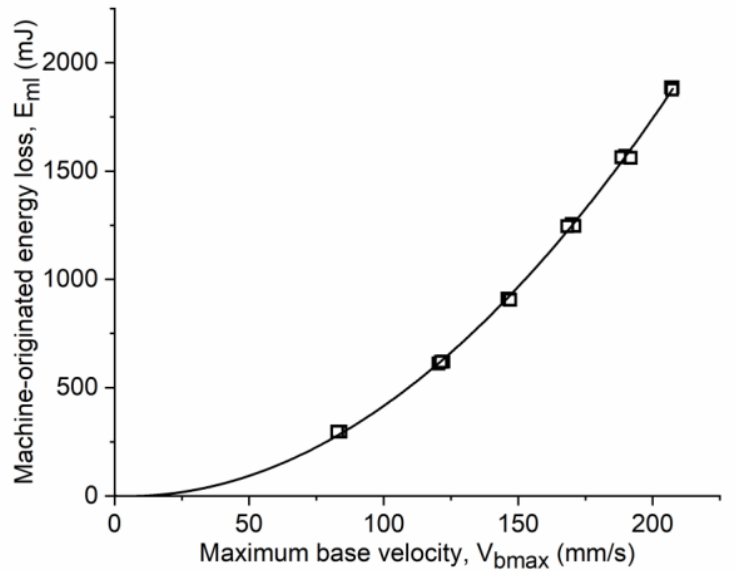

736

737

738

739 
Fig. 3

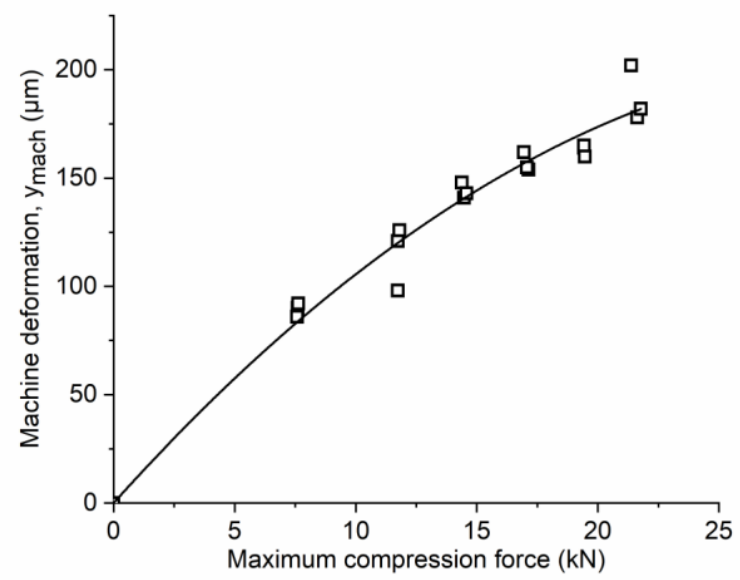

Fig. 4
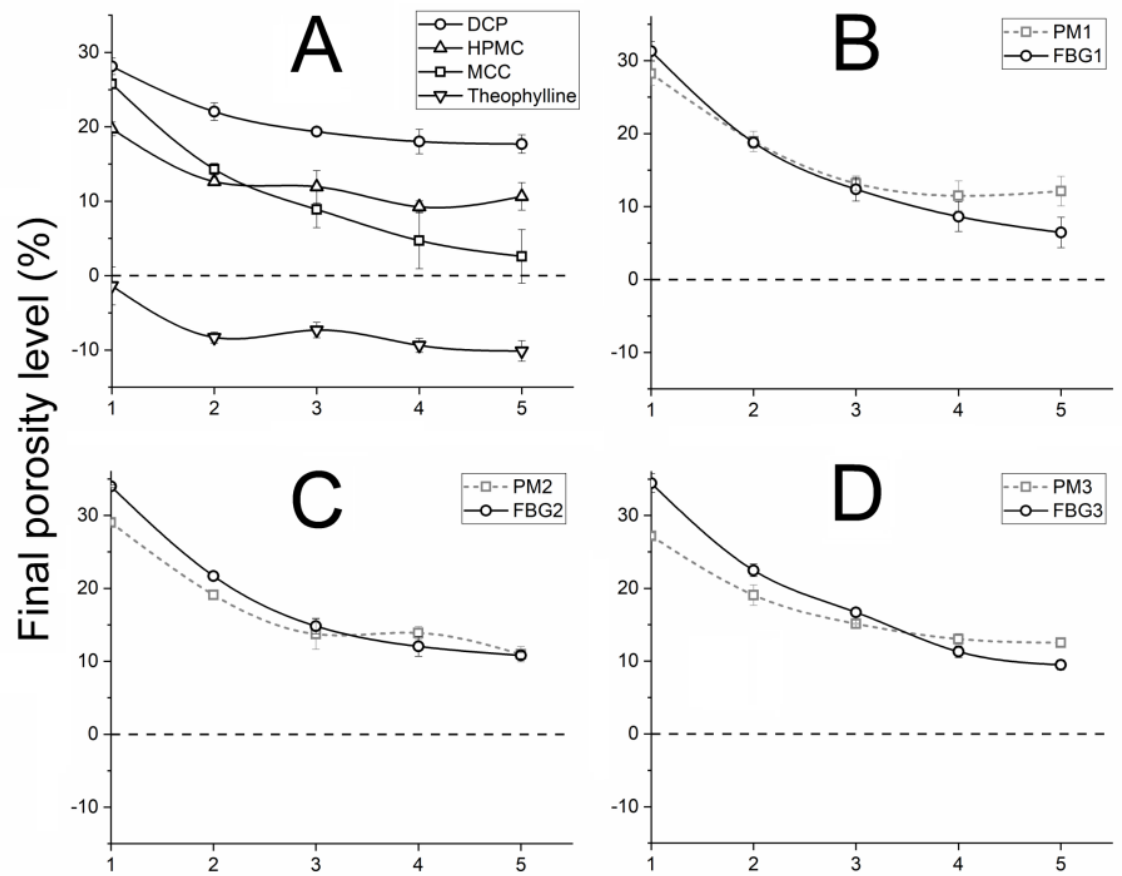

Compression no 
Fig. 5

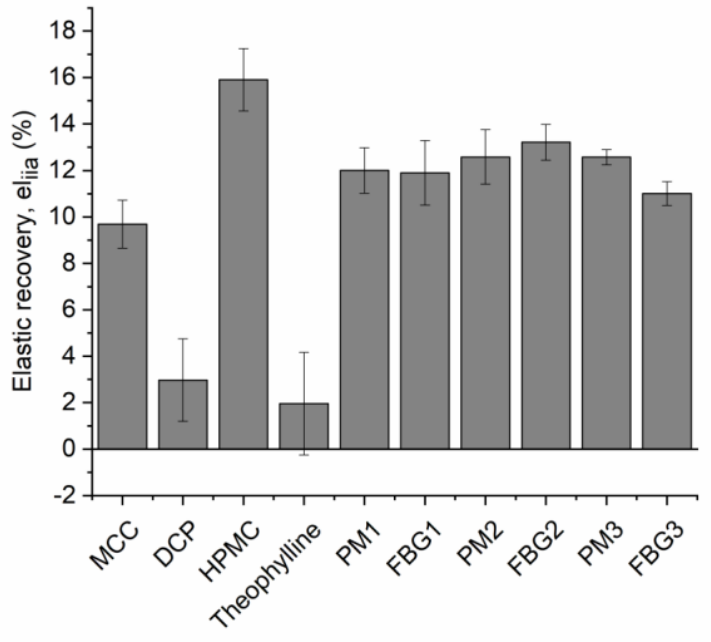

$751 \quad$ Fig. 6

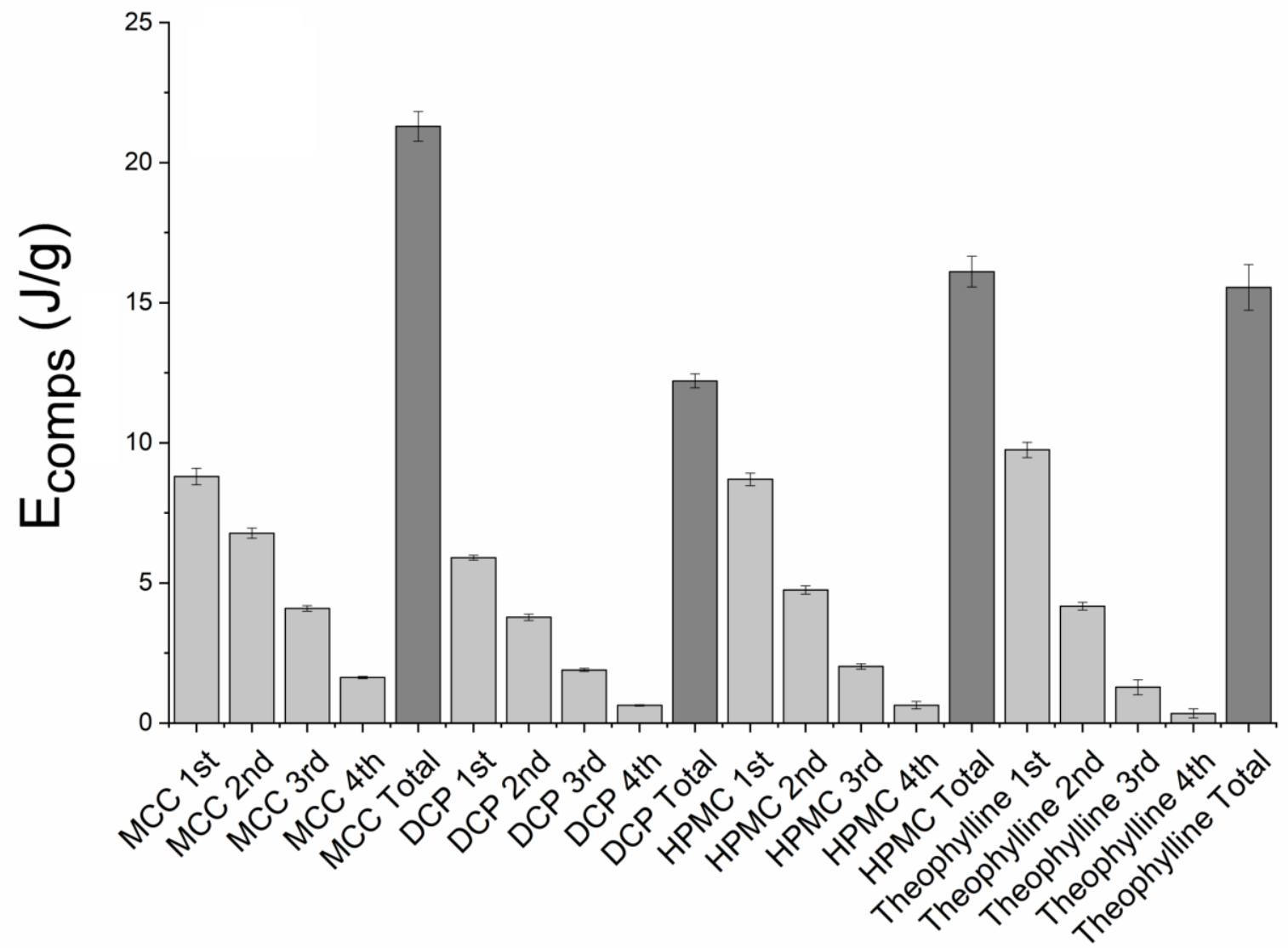


Fig. 7

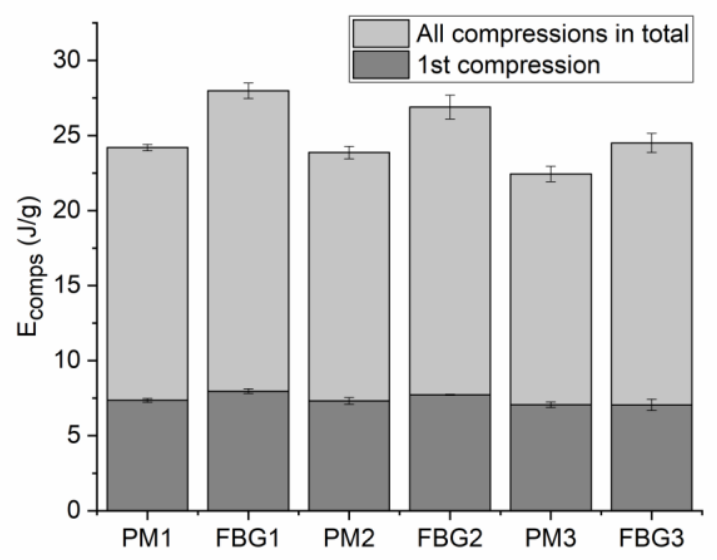

755

756

$757 \quad$ Fig. 8

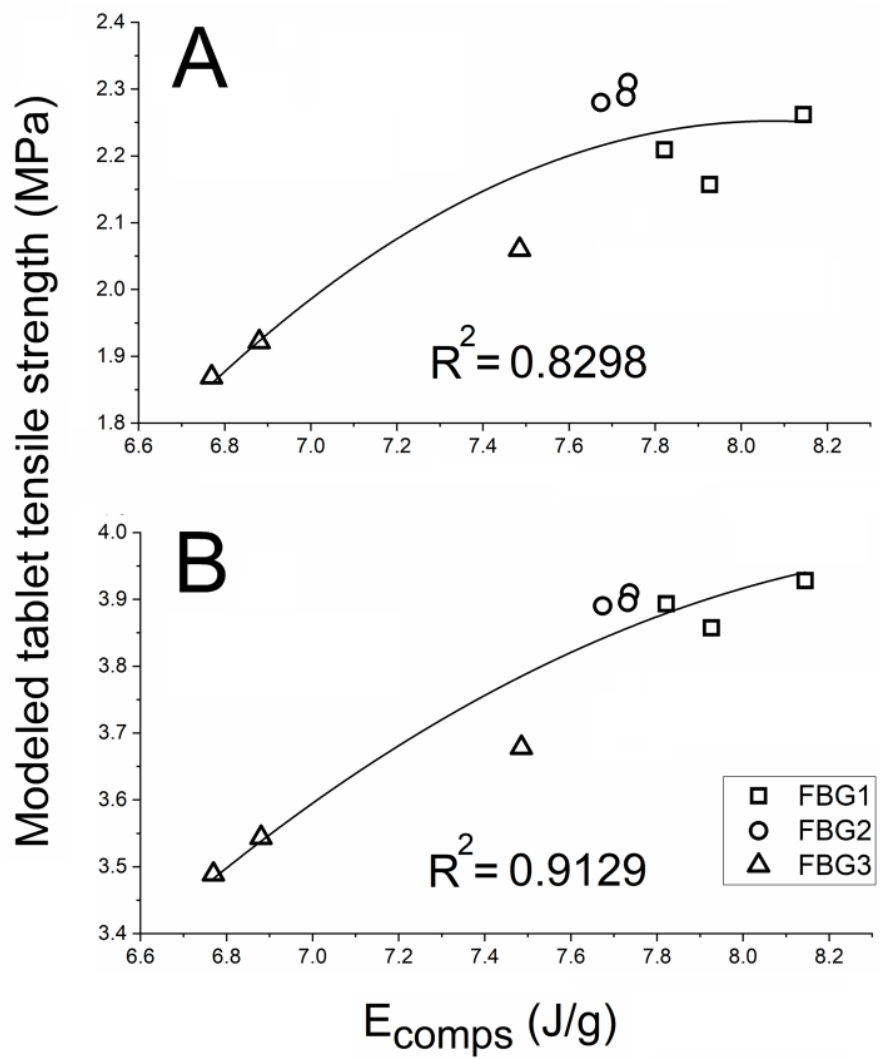

